Original Article

\title{
PREVALENCE AND ANTIBIOTIC SUSCEPTIBILITY OF ACINETOBACTERIN A TERTIARY CARE HOSPITAL IN CHENNAI, INDIA
}

\author{
ANGELINE ANJALI A. ${ }^{1}$, ABIRAMASUNDARI V. K. ${ }^{2}$ \\ ${ }^{1}$ Saveetha Medical College and Hospital, Chennai, ${ }^{2}$ Department of Microbiology, Saveetha Medical College and Hospital \\ Email: angelineanjali03@gmail.com
}

Received: 14 Sep 2020, Revised and Accepted: 18 Nov 2020

\section{ABSTRACT}

Objective: The present study is to determine the prevalence and antibiotic susceptibility of Acinetobacter species in samples collected from patients in tertiary care hospital in Chennai.

Methods: A total of17,827patient's clinical samples were collected from various wards and ICUs of Saveetha Medical College and Hospital, Chennai, Tamilnadu over a period of 7 mo [between January 2020 and July 2020]. All samples were tested in the microbiology lab of Saveetha Medical College and Hospital using standard operating procedures.

Results: Out of 17,827 samples, 2,816 were culture positive. 122 of the isolates tested positive for Acinetobacter spp.and $81.1 \%$ of the isolates belonged to Acinetobacterbaumannii. Most of the infection occurred in the age group of 21-40 y and predominantly in female patients (female, male ratio 1.9:1).General wards contributed to $54.9 \%$ of the Acinetobacter infection, followed by ICU(27\%) and OPD(18\%). Maximum isolates were recovered from urine(34.4\%) and endotracheal secretions(29.5\%).60.7\% of the Acinetobacterspp were multidrug-resistant(MDR)i.e. resistant to more than 3 antibiotic group.In our study, most Acinetobactersppwere resistant to penicillin(46-100\%), third and fourth generation cephalosporin (36-61.5\%), carbapenems (34.4-82.8\%)and quinolones(39.3-46.7\%). None of the isolates were resistant to colistin. 93.4\% ofisolates were sensitive to tigecycline and $87.7 \%$ sensitive to amikacin.

Conclusion: Our study observed a high incidence of MDR inAcinetobacterspp, which is in line with most of the research findings in recent times. Most of Acinetobacterspp were resistant to penicillin, third and fourth generation cephalosporins, quinolones, carbapenems,which is alarming as it leaves fewer options for the line of treatment. Some strains were sensitive to cefepime, ceftazidime, piperacillin-tazobactam, levofloxacin, imipenem and meropenem. Considering the increasing MDR nature of Acinetobacterspp a combination of the former along with colistin, tigecycline, amikacin(which have shown more than $85 \%$ sensitivity) would need to be studied.Also, strict measures to control the spread of Acinetobacter infection, better management of antibiotics usage and newer therapeutic option for treatment need to be looked at.

Keywords: Acinetobacter, Multidrug-resistant,Prevalence, Antibiotics,Resistance, Sensitive, Carbapenem, Cephalosporin, Quinolones, Infection, Hospital-acquired infection

(C) 2021 The Authors. Published by Innovare Academic Sciences Pvt Ltd. This is an open access article under the CC BY license (https://creativecommons.org/licenses/by/4.0/) DOI: https://dx.doi.org/10.22159/ijcpr.2021v13i1.40803. Journal homepage: https://innovareacademics.in/journals/index.php/ijcpr

\section{INTRODUCTION}

Acinetobacter is a group of gram-negative coccobacilli that are nonmotile, strictly aerobic, catalase-positive, and oxidase-negative, which are commonly found in the environment, like soil and water[1]. In humans, Acinetobacter can colonize in skin, wounds, and the respiratory and gastrointestinal tracts. It can cause serious conditions like sepsis, meningitis, pneumonia, necrotizing fasciitis [2]. It has become a pathogen of increasing significance because over the decades it has grown tobecome a major cause of hospital acquired infection, a major problem confronting ICU clinicians [3]. One of the reasons is due its ability to survive for long periods on hospital surface and equipment. Also,Acinetobacterspp has the extraordinary ability to develop multiple resistance against major antibiotic classes which has made it even more difficult to treat the infection. They have become highly resistant to broad spectrum of antibiotics like penicillin, third-generation cephalosporins, carboxypenicillins, carbapenems [4]. Most strains are resistant to fluoroquinolones as well.Acinetobacterspp produce a wide range of aminoglycoside-inactivating enzymes which is also one of the causes for increased resistance to antibiotics [5].There is a significant difference in the behavior and spread of multi-drug resistant Acinetobacter spp. recovered in various geographic locations. As several factors cause resistance in Acinetobacterspp, treatment of infections caused by this organism should be based on antibiotic susceptibility tests. Therefore, having information regarding the prevalence and pattern of bacterial resistance to these drugs is important. Keeping these above facts in view, we have analyzed the frequency, risk factors, and resistance pattern of Acinetobacter spp. that were isolated from different clinical samples in a tertiary care hospital in Chennai, India.

\section{MATERIALS AND METHODS}

A retrospective, hospital record-based, cross-sectional study was carried out from Jan 2020 to July 2020 in the Department of Clinical Microbiology at a tertiary care hospital in Chennai. A total of 17,827 clinical samples like pus/swab, urine, sputum, blood, body fluid, tracheal aspirate, endotracheal tube, and intravenous(IV) catheter tips were collected from the patients and transferred to the laboratory without delay for further processing. The study was conducted after due approval was obtained from the institutional ethical committee

\section{Sample processing and antibiogram}

In the laboratory, all the collected samples were cultured aerobically on blood agar and MacConkey agar. Blood specimen was cultured in trypticase soy broth (TSB) and subcultured in blood agar and chocolate agar. The isolation,identification, and speciation were done according to the standard procedure [5]. All the isolates were tested for antimicrobial susceptibility testing by the standard Kirby-Bauer disk diffusion method. Samples were processed for culture by standard conventional methods and susceptibility testing were determined by Kirby Bauers disc diffusion method. Antibiotics and their strength used was according to the Clinical and Laboratory Standards Institute (CLSI) guidelines [6].

\section{RESULTS}

During the period of study from Jan 2020 to July 2020 a total of 17,827 samples were examined from different age group, admitted 
in various departments of the hospital. Of the total samples processed, 2,816 samples were culture positive and showed growth of different microorganism. Out of these positive isolates, 122(4.3\%) were confirmed as Acinetobacterspp as represented in fig. 1.

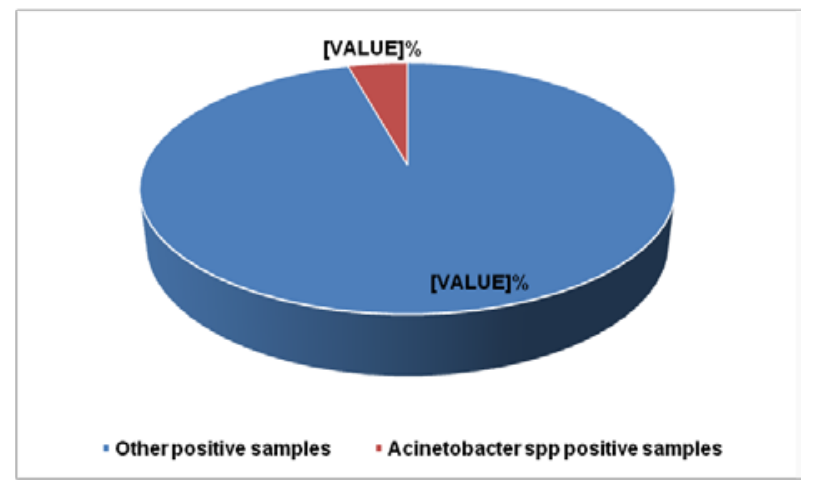

Fig.1: Percentage of acinetobacterspp isolates in total positive samples

Of the total 122 Acinetobacterspp isolates, 99(81.1\%) were Acinetobacterbaumannii. Other species included Acinetobacterjunii $9(7.4 \%)$, Acinetobacterlowffii
Acinetobactercalcoaceticus4(3.3\%) and Acinetobatcerursingii and haemolyticus together $5(4.1 \%)$ isolates. Fig. 2 shows the distribution of the various Acinetobacter spp.

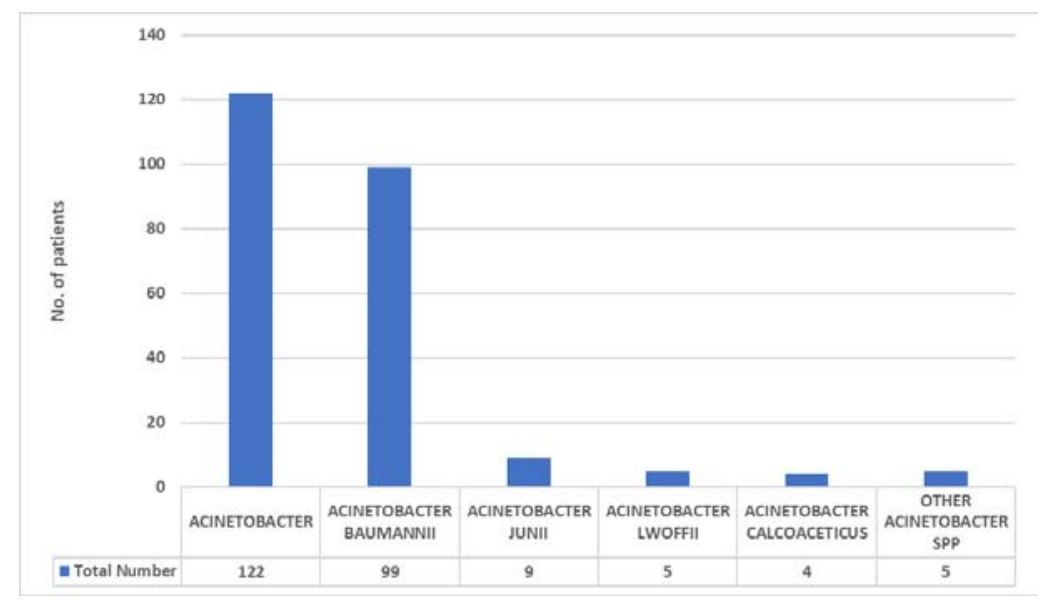

Fig.2: Species-wise distribution of Acinetobacter isolates

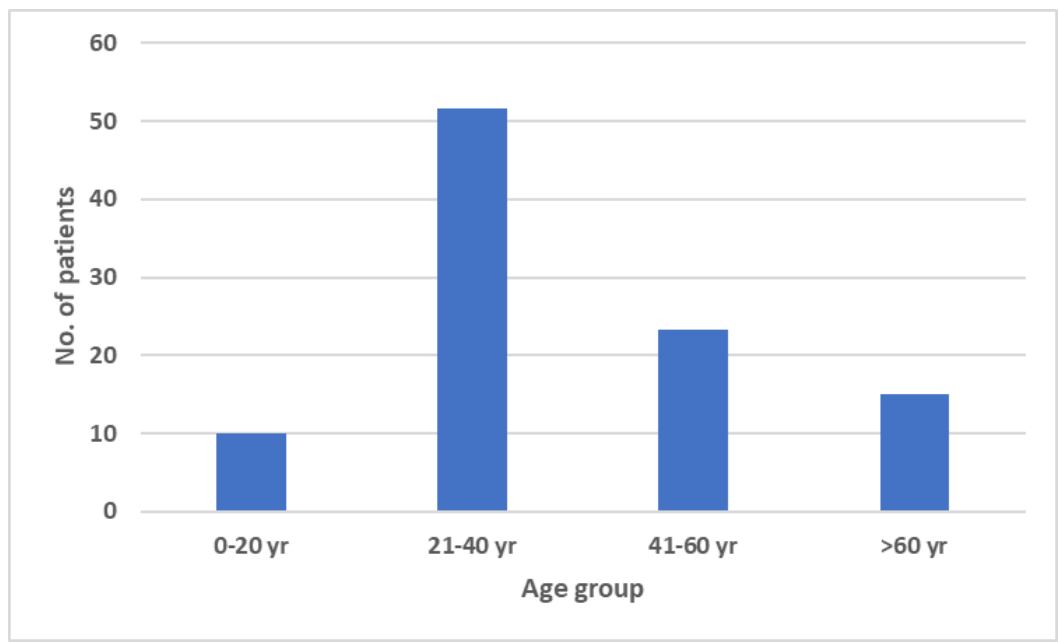

Fig.3: Age-wise distribution of Acinetobacterisolates 


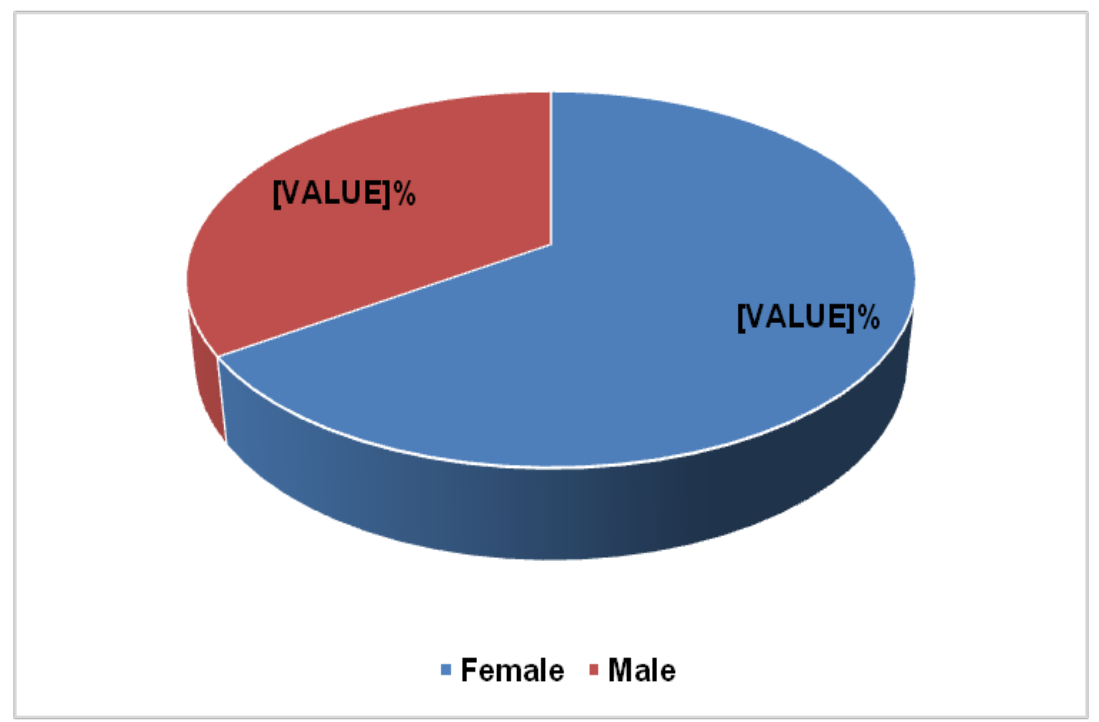

Fig.4: Gender-wise distribution of Acinetobacterisolates

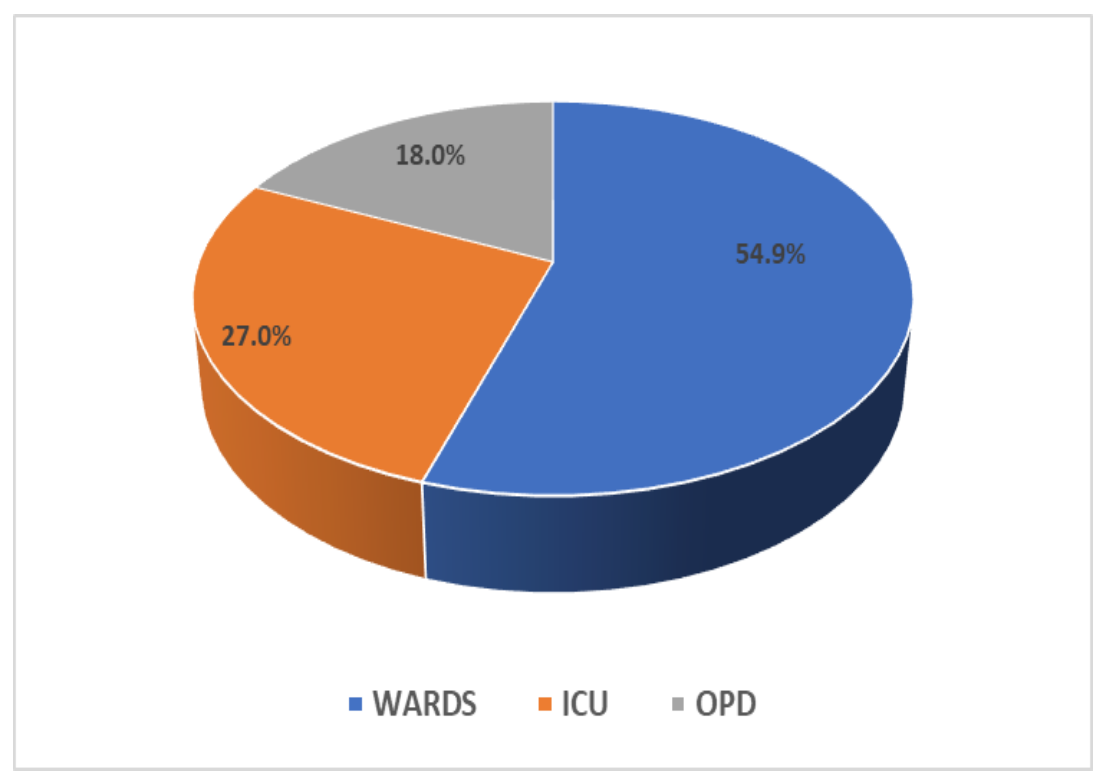

Fig.5: Distribution of isolates from Wards, ICU, OPD

Table 1: Distribution of study isolates in various specimens

\begin{tabular}{ll}
\hline Clinical samples & No. of isolates (\%) \\
\hline Urine & $42(34.4 \%)$ \\
Endotracheal & $36(29.5 \%)$ \\
Blood & $22(18.0 \%)$ \\
Exudate & $16(13.1 \%)$ \\
Pleural Fluid & $3(2.5 \%)$ \\
Sputum & $2(1.6 \%)$ \\
BAL & $1(0.8 \%)$ \\
\hline
\end{tabular}


Table 2: Antibiotics susceptibility patter in the acinetobacterstudy isolates

\begin{tabular}{lll}
\hline Total acinetobacterisolates $(\mathbf{n}) \mathbf{~ 1 2 2}$ & & \\
\hline Antibiotics & Resistant isolates & Sensitive isolates \\
\hline Ampicillin & $122(100 \%)$ & $0(0 \%)$ \\
Piperacillintazobactam & $56(45.9 \%)$ & $66(54.1 \%)$ \\
Gentamicin & $64(52.5 \%)$ & $59(47.5 \%)$ \\
Amikacin & $15(12.3 \%)$ & $107(87.7 \%)$ \\
Cefoperazonesulbactam & $55(45.1 \%)$ & $67(54.9 \%)$ \\
Ceftriaxone & $75(61.5 \%)$ & $47(38.5 \%)$ \\
Ceftazidime & $44(36.1 \%)$ & $78(63.9 \%)$ \\
Cefepime & $53(43.4 \%)$ & $69(56.6 \%)$ \\
Ciprofloxacin & $57(46.7 \%)$ & $65(53.3 \%)$ \\
Levofloxacin & $48(39.3 \%)$ & $74(60.7 \%)$ \\
Cotrimoxazole & $61(50.0 \%)$ & $61(50.0 \%)$ \\
Ertapenem & $101(82.8 \%)$ & $21(17.2 \%)$ \\
Imipenem & $48(39.3 \%)$ & $74(60.7 \%)$ \\
Meropenem & $42(34.4 \%)$ & $80(65.6 \%)$ \\
Nitrofurantoin & $107(87.7 \%)$ & $15(12.3 \%)$ \\
Tigecycline & $8(6.6 \%)$ & $114(93.4 \%)$ \\
Colistin & $0(0 \%)$ & $122(100 \%)$ \\
\hline
\end{tabular}

As observed in table 2, none of the isolates were resistant to colistin. Other sensitive drugs were tigecycline $114(93.4 \%)$ and amikacin $107(87.7 \%)$.

Acinetobacter species showed high resistance to ampicillin (100\%), nitrofurantoin 107 (87.7\%), ertapenem 101(82.8\%), ceftriaxone $75(61.5 \%)$, gentamicin $64(52.5 \%)$, cotrimoxazole 61(50.0\%), ciprofloxacin $\quad 57(46.7 \%)$,piperacillin- tazobactam56(45.9\%), cefoperazonesulbactam 55(45.1\%), cefepime 53(43.4\%).

Most of the 122 positive Acinetobacter. spp were resistant to multiple antibiotics as seen in the table 3 below.

Table 3: MDR distribution in the study isolates

\begin{tabular}{ll}
\hline Isolates resistant to 'n'of antibiotic groups & Resistant No. \\
\hline $\mathrm{n}>2$ & $80(65.6 \%)$ \\
$\mathrm{n}>3$ & $74(60.7 \%)$ \\
$\mathrm{n}>4$ & $65(53.3 \%)$ \\
$\mathrm{n}>5$ & $54(44.3 \%)$ \\
\hline
\end{tabular}

More than $60.7 \%$ of the 122 Acinetobacter positive isolates were resistant to more than 3 classesof antibiotics and were classified as MDR.

\section{DISCUSSION}

In the present study, from the 2,816 isolates, $122(4.3 \%)$ of the isolates tested positive for Acinetobacter spp. Similar prevalence of $4.5 \%$ and $3.4 \%$ of the total organism isolated was reported by Rit $\mathrm{K}$. et al. in Odisha and Gupta et al. in Pune $[7,8]$.

Among the Acinetobacterspp,99(81.1\%) of the strains were confirmed as Acinetobacterbaumannii and remaining 23(18.9\%) as other Acinetobacter species(fig. 2). Study by Sharma et al.[9] also reported a similar value of $230(83.3 \%)$ positive Acinetobacterbaumannii, while Rit K. et al.[7] reported aslightly lower incidence of $74.02 \%$. Only a study in West Bengal in 2012 by Bhattacharyya. et al. reported a very low value of $54 \%$ baumannii species[10].

Most infections occurred in age group 21-40 y (52) followed by 4160 age group(23) (fig. 3). Similar prevalence was reported by most studies $[9,11,12]$.

Gender ratio was 1.9:1, which shows a female preponderance in our study. This was not the case with most studies that reported a slight male preponderance [7-9]. Saha et al. and Sivaranjaniet al.[11,12] have reported slight predominance of female patients over male. One reason for was predominance of female in our study was the high number of female in-patients in obstetrics wards, which accounted for $20 \%$ of the isolates.

In the present study maximum isolates were isolated from general wards 67(54.9\%) followed by ICU 33(27\%). Refer fig. 5 above. Most Acinetobacterspp studies have pointed to high incidence of Acinetobacter infection in patients admitted in hospital especially in ICUsas they are capable of rapid adaptation to the hospital environment[13-15].

Maximum number of isolates were from urine specimen $42(34.4 \%)$ followed by endotracheal 36(29.5\%), 22(18\%) in blood and exudate 16(13.1\%). Bhattacharyya et al.[10] also reported maximum isolates from urine samples (54\%). While,Shanthiet al.[16]reporteda maximum number of isolates from endotracheal (ET) secretion $(41.8 \%)$, followed by urinary tract $(25.5 \%)$, wound $(20 \%)$ and blood (12.7\%). Sharma et al.[9] and Jaggiet al.[14]also reported maximum isolates from respiratory secretions.

Out of the 122 isolates, 74(60.7\%) isolates were Multi-Drug Resistant (MDR), defined as resistance to $>3$ antimicrobial agent groups. This is the similar finding of $62.1 \%$ reported by Saha $S$ etal.[11] in 2018. While the study in USA and Puerto Rico[17] reported MDR in 54\% of its Acinetobacter isolates in 2012.

Our findings on resistance to the commonly prescribed cephalosporins namely ceftazidime and cefepime (36.1\%-43.4\%) are consistent with results from several previous studies in the Netherlands[18], USA[17] and India[19], which reported resistance rate of $16 \%-56 \%$ to ceftazidime and cefepime. Studies in Pakistan[20] have reported very high resistance to ceftazidime and cefepime (both $99.2 \%$ ), which could be due to extensive use of antibiotics. A 55(45.1\%) resistance to cefoperazonesulbactam was also recorded in our study, which is similar to $44.78 \%$ resistance reported by Saderet al. (46.8\%)[21] and Lai CC et al. (40.0\%)[22].

Resistance towards carbapenems like imipenem, meropenem was recorded to be $48(39.3 \%)$ and $42(34.4 \%)$, respectively. A lower resistance to imipenem (25.3\%) and meropenem (29.7\%) was reported by Saha et al.[11].

Studies as early as 1996 show that carbapenems were highly 
sensitive to Acinetobacterspp and were used as first line drug of choice to treat Acinetobacter infection[15,19,23].Data collected from 37 centers in 11 European countries between 1997 and 2000 reported imipenem and meropenem as the most active agents against Acinetobacter, with resistance rates of $16 \%$ and $18 \%$ respectively[24]. However, the subsequent data from 12 European countries18 revealed a significant increase in the resistance rates against imipenem (42.5\%) and meropenem (43.4\%)[25].Data of the antibiotic susceptibilities from different geographical regions revealed that the resistance of Acinetobacter spp. to imipenem was in the range of, no resistance to $40 \%$ (2000-2004)[26].Other studies have also reported increasing resistance to carbapenems[9,23],which is reflected in our study as well.

Penicillin group of antibiotics showed high resistance to Acinetobacter spp. Ampicillin showed maximum resistance and for piperacillintazobactam 56(45.9\%). Slightly higher value of resistance for piperacillin-tazobactam $50.5 \%$ has been reported by Saha et al.[11]

Aminoglycosides resistance was observed with $64(52.5 \%)$ isolates resistant to gentamicin while Acinetobacter showed more sensitivity to amikacin $107(87.8 \%)$. Most studies[7,11] have shown similar high sensitivity(75\%-85\%) to amikacin and therefore it is considered as one of the drugs of choice in combination with others for treating Acinetobacterspp[27].

None of the isolates were resistant to colistin. Most of the Acinetobacterspp sensitivity study in India and US $[9,11,12,14,28,29]$ have reported mostly zero or around $1 \%$ resistance to colistin.Studies in other countries have however shown increase in colistin resistance. Study in Western Pacific[30] region showed $3.3 \%$ resistance to colistin. Colistin resistance rates of Acinetobacterspp strains isolated in Germany[31] were 2.8\%.In Korea[32], there was high resistance to colistin (30.6\%).However, as the resistance against colistin is not very high in our country it can be still be used in case of MDR Acinetobacter spp.

In our study, there was $6.6 \%$ resistance to tigecycline. Study in Germany[31]also reported $6 \%$ resistance. A study in Turkey reported considerably higher tigecycline resistance rates $(25 \%)$ forAcinetobacter strains[33].

Sensitivity to colistin was $100 \%$ followed by tigecycline $114(93.4 \%)$ followed by Amikacin 107(87.7\%)

Fluoroquinolone, namely ciprofloxacin, levofloxacin showed around 40\%-50\% resistance to Acinetobacter spp. Which was similar to data reported by Saha et al.[11]Fluoroquinolones have never been proved to be a mainstay antibiotic for Acinetobacterspp due to increased resistance over the years. However, usage of levofloxacin, along with colistin has shown better results[34].

\section{CONCLUSION}

Our study observed a high incidence of MDR in Acinetobacterspp, which is in line with most research in recent times. Most of them were resistant to penicillin, third and fourth generation cephalosporins, quinolones, carbapenems, which is alarming as it leaves fewer options for line of treatment. Some strains were sensitive to cefepime, ceftazidime, piperacillin-tazobactam, levofloxacin, imipenem and meropenem. Considering the increasing MDR nature of Acinetobacterspp a combination of the former along with colistin, tigecycline, amikacin(which have shown more than $85 \%$ sensitivity) would need to be studied. Also, strict measures to control the spread of Acinetobacter infection, better management of antibiotics usage and newer therapeutic option for treatment need to be looked at.

\section{ACKNOWLEDGEMENT}

To microbiology department of Saveetha medical college and hospital for providing data of patient samples.

\section{FUNDING}

Nil

\section{AUTHORS CONTRIBUTIONS}

All the authors have contributed equally.

\section{CONFLICTS OF INTERESTS}

\section{Declared none}

\section{REFERENCES}

1. EBergogneBerezin. Bacteria: Acinetobacter. Encyclopedia Food Safety 2014;1:337-41.

2. Michael J McConnell, Luis Actis, JeronimoPachon.Acinetobacterbaumannii: human infections, factors contributing to pathogenesis and animal models. FEMS Microbiol Rev 2013;37:130-55.

3. Uwingabiye J, Lemnouer A, Baidoo S. Intensive care unitacquired Acinetobacterbaumannii infections in a moroccan teaching hospital: epidemiology, risk factors and outcome. Germs 2017;7:193-205.

4. Manchanda V, Sanchaita S, Singh N. Multidrug resistant acinetobacter. J Glob Infect Dis 2010;2:291-304.

5. Collee JG, Miles RS, Watt B. Tests for the identification of bacteria. In: Collee JG, Fraser AG, Marmion BP, Simmons A. (eds). Mackie and McCartney practical medical microbiology. $14^{\text {th }}$ edn. Edinburgh: Churchill; 1996. p. 131-51.

6. Clinical and Laboratory Standards Institute. Performance standards for antimicrobial susceptibility testing: 20th informational supplement. CLSI document M100-S20. Wayne, Pennsylvania: Clinical and Laboratory; 2020.

7. Rit K, Saha R. Multidrug-resistant acinetobacter infection and their susceptibility patterns in a tertiary care hospital. Niger Med J 2012;52:126-8.

8. Gupta N, Gandham N, Jadhav S, Mishra RN. Isolation and identification of Acinetobacterspecies with special reference to antibiotic resistance. J Nat SciBiol Med 2015;6:159-62.

9. Sharma RK, Mamoria VP. A prospective study on prevalence and antibiotic susceptibility pattern of Acinetobacterbaumannii in clinical samples obtained from patients admitted in various wards and intensive care units. J Mahatma GandhiUniv Med Sci Tech 2017;2:122-7.

10. Bhattacharyya S, Bhattacharyya I, Rit K, Mukhopadhyay PK, Dey JB, Ganguly U, et al. Antibiogram of Acinetobacter spp. isolated from various clinical specimens in a tertiary care hospital in West Bengal, India. Biomed Res 2013;24:43-6.

11. Saha S, Devi KM, Damrolien S, Devi KS. A study of acinetobacter infection in a tertiary care hospital in Northeast India. Int J Res Med Sci 2018;6:2076-80.

12. Sivaranjani V, Umadevi S, Srirangaraj S, Kali A, Seetha KS. Multidrug resistant Acinetobacter species from various clinical samples in a tertiary care hospital from South India. AMJ 2013;6:12, 697-700.

13. E BergogneBerezin, $\mathrm{K} J$ Towner.Acinetobacter spp. as nosocomial pathogens: microbiological, clinical, and epidemiological features. ClinMicrobiol Rev 1996;9:148-65.

14. Jagii N, Sissodia P, Sharma L.Acinetobacterbaumannii isolates in a tertiary care hospital: antimicrobial resistance and clinical significance. J Microbiol Infectious Diseases 2012;2:57-63.

15. Alişkan H, Colakoglu $S$, Turunç T. Four years of monitoring of antibiotic sensitivity rates of Pseudomonas aeruginosa and acinetobacterbaumannii strains isolated from patients in intensive care unit and inpatient clinics. MikrobiyolBul 2008;42:321-9.

16. Shanthi M, Sekar U. Multi-drug resistant pseudomonas aeruginosa and Acinetobacterbaumannii infections among hospitalized patients: risk factors and outcomes. J Assoc Physicians India 2009;8:687-93.

17. Queenan AM, Pillar CM, Deane J. Multidrug resistance among Acinetobacter spp. in the USA and activity profile of key agents: results from capital surveillance 2010. DiagnMicrobiol Infect Dis 2012;73:267-70.

18. Lazureanu V, Porosnicu M, Gandac C, Moisil T, Baditoiu L, Laza $\mathrm{R}$, et al. Infection with Acinetobacterbaumannii in an intensive care unit in the Western part of Romania. BMC Infect Dis 2016;(16 Suppl 1):95.

19. Broek PJ, Van Der Reijden TJK, Van Strijen E, HelmigSchurter AV, Bernards AT, Dijkshoorn L. Endemic and epidemic 
Acinetobacter species in a university hospital: an 8 y survey. J ClinMicrobiol 2009;47:3593-9.

20. Sohail M, Rashid A, Aslam B, Waseem M, Shahid M, Akram M, et al. Antimicrobial susceptibility of Acinetobacterclinical isolates and emerging antibiogram trends for nosocomial infection management. Rev Soc Bras Med Trop 2016;49:300-4.

21. Sader SH, Carvalhaes GC, Streit MG, Castanheira M, Flamm KR Antimicrobial activity of cefoperazone-sulbactam tested against gram-negative organisms from Europe, Asia-Pacific, and Latin America. IntJ Infectious Diseases 2020;91:32-7.

22. Lai CC, Chen CC, Lu YC, Chuang YC, Tang HJ. In vitro activity of cefoperazone and cefoperazone-sulbactam against carbapenem-resistant Acinetobacterbaumannii and pseudomonas aeruginosa. Infect Drug Resist 2018;12:25-9.

23. Turk Dagi $\mathrm{H}$, Kus H, Arslan U, Tuncer I. In vitro synergistic activity of sulbactam in combination with imipenem, meropenem and cefoperazone against carbapenem-resistant Acinetobacterbaumannii isolates. MikrobiyolBul 2014;48:311-5.

24. Turner PJ, Greenhalgh JM. MYSTIC study group (Europe) the activity of meropenem and comparators against Acinetobacter strains isolated from European hospitals, 1997-2000. ClinMicrobiol Infect 2003;9:563-7.

25. Turner PJ. Meropenem activity against European isolates: report on the MYSTIC (Meropenemyearly susceptibility test information collection) 2006 results. DiagnMicrobiol Infect Dis 2008;60:185-92.

26. Perez F, Hujer AM, Hujer KM, Decker BK, Rather PN, Bonomo RA. Global challenge of multidrug-resistant Acinetobacterbaumannii. Antimicrob Agents Chemother2007;51:3471-84

27. Joel Fishbain, Anton Y Peleg. Treatment of acinetobacter infections.Clin Infectious Diseases 2010;51:79-84.
28. Dash M, Padhi S, Patnaik S, Mohanty I, Misra P. Frequency, risk factors, and antibiogram of Acinetobacter species isolated from various clinical samples in a tertiary care hospital in Odisha, India. Avicenna J Med 2013;3:97-102.

29. Adams Haduch JM, Paterson DL, Sidjabat HE. Genetic basis of multidrug resistance in Acinetobacterbaumannii clinical isolates at a tertiary medical center in Pennsylvania. Antimicrob Agents Chemother 2008;52:3837-43.

30. Yau W, Owen RJ, Poudyal A.Colistinheteroresistance in multidrug-resistant Acinetobacterbaumannii clinical isolates from the western pacific region in the SENTRY antimicrobial surveillance programme. J Infect 2009;58:138-44.

31. Seifert H, Stefanik D, Wisplinghoff $H$. Comparative in vitro activities of tigecycline and 11 other antimicrobial agents against 215 epidemiologically defined multidrug-resistant Acinetobacterbaumannii isolates. J AntimicrobChemother 2006;58:1099-100.

32. KnamSooKo, JiYoeunSuh, Ki Tae Kwon. High rates of resistance to colistin and polymixin $B$ in subgroups of Acinetobacterbaumannii isolates from Korea. J AntimicrobChemother 2007;60:1163-7.

33. Dizbay M, Altuncekic A, Sezer BE, Ozdemir K, Arman D. Colistin and tigecycline susceptibility among multidrug-resistant Acinetobacterbaumannii isolated from ventilator-associated pneumonia. Int J Antimicrob Agents 2008;32:29-32.

34. Wei $\mathrm{W}$, Yang $\mathrm{H}, \mathrm{Hu}$ Lifen, Ye Ying,Li Jiabin. Activity of levofloxacin in combination with colistin against Acinetobacterbaumannii: in vitro and in a galleria mellonella model. J MicrobiolImmunol Infection 2017;50:821-30. 